\title{
Le licenciement des salariés pour perte de confiance
}

\author{
Maria Katia Garcia Landaburu
}

\section{(2) OpenEdition}

1 Journals

\section{Édition électronique}

URL : https://journals.openedition.org/rdctss/1683

DOI : $10.4000 /$ rdctss. 1683

ISSN : 2262-9815

Éditeur

Centre de droit comparé du travail et de la sécurité sociale

\section{Édition imprimée}

Date de publication : 1 avril 2019

Pagination : 190-193

ISSN : 2117-4350

\section{Référence électronique}

Maria Katia Garcia Landaburu, « Le licenciement des salariés pour perte de confiance », Revue de droit comparé du travail et de la sécurité sociale [En ligne], 1 | 2019, mis en ligne le 01 novembre 2021, consulté le 13 novembre 2021. URL : http://journals.openedition.org/rdctss/1683 ; DOI : https:// doi.org/10.4000/rdctss. 1683

\section{(c) (i) $\odot$}

Revue de droit comparé du travail et de la sécurité sociale est mise à disposition selon les termes de la Licence Creative Commons Attribution - Pas d'Utilisation Commerciale - Pas de Modification 4.0 International. 


\title{
MARIA KATIA GARCIA LANDABURU
}

\author{
Université PONTIFICALE CATHOLIQUE du PÉrou
}

\section{LE LICENCIEMENT DES SALARIÉS POUR PERTE DE CONFIANCE}

Le 1er septembre 2018, les accords adoptés en matière de travail lors de la VIIème Séance plénière des juridictions suprêmes ont été publiés au journal officiel «El Peruano ». Les Juges suprêmes de la Première et de la Deuxième Chambres de droit constitutionnel et de droit social de la Cour suprême de justice de la République ont participé à cette séance plénière, décidant à l'unanimité que les salariés de direction ou de confiance ${ }^{1}$ des entreprises et des institutions du secteur privé auxquels l'employeur retire sa confiance ont droit à ce qui suit:

- Un salarié qui a directement accédé à un poste de confiance ou de direction n'a pas droit au versement d'indemnité pour licenciement arbitraire.

- Un salarié qui a occupé un poste avec des fonctions communes ou ordinaires, et qui par la suite a accédé à un poste de confiance ou de direction au sein de la même entreprise ou institution privée, a droit au versement d'indemnités pour licenciement arbitraire lorsque son employeur l'empêche de réintégrer l'ancien poste de travail à la suite d'une perte de confiance ou lorsque le salarié lui-même choisit de ne pas réintégrer son ancien poste de travail.

Ces dispositions sont discutables car elles laissent sans protection les salariés de direction ou de confiance qui, occupant de tels postes dès le début de leur relation de travail, sont tributaires de ce que leur employeur pourra décider de nommer "perte de confiance", hypothèse non prévue dans la législation sur les motifs légitimes de licenciement.

\section{I - LE RÉGIME LÉGAL DE LA SÉCURITÉ DE L'EMPLOI}

La Constitution du Pérou de 1993 prévoit que le travail est un droit et un devoir (article 22), aucune relation de travail ne pouvant limiter l'exercice des droits constitutionnels, ni ignorer ou porter atteinte à la dignité du salarié (article 23). Pour ce qui est de la protection du salarié face au licenciement arbitraire, la Constitution établit que « la loi accorde au salarié une protection adéquate contre le licenciement arbitraire » (article 27).

1 En vertu de l'article 43 de la loi sur la productivité et la compétitivité du travail, le personnel de direction « est celui qui exerce la représentation générale de l'employeur face aux autres salariés ou à des tiers, ou qui le remplace, ou qui partage avec lui certaines fonctions d'administration et de contrôle, ou dont l'activité et le niveau de responsabilité influencent le résultat de l'activité de l'entreprise ». Ce même texte définit les salariés de confiance comme ceux «qui travaillent en contact personnel et direct avec l'employeur ou avec le personnel de direction, ont accès à des secrets industriels, commerciaux ou professionnels et, de façon générale, à des informations sensibles. II s'agit aussi de ceux dont les opinions ou rapports sont directement présentés au personnel de direction et qui contribuent ainsi aux décisions de l'entreprise ». 
Le texte unique du décret législatif $n^{\circ} 728$, Loi sur la productivité et la compétitivité du travail, approuvé par le décret suprême $n^{\circ}$ 003-97-TR (ci-après LPCT), dispose à l'article 22 que, pour le licenciement d'un salarié du secteur privé, qui travaille quatre heures ou plus par jour pour le même employeur, l'existence d'un motif légitime prévu par la loi et dûment contrôlé est indispensable.

Conformément à l'article 34 de la LPCT, le licenciement du salarié fondé sur des motifs liés à son comportement ou à ses compétences ne donne pas lieu à indemnisation. Si le licenciement est arbitraire parce qu'aucun motif n'a été invoqué ou parce que le motif invoqué n'a pas pu être prouvé devant un tribunal, le salarié a droit au paiement des indemnités de licenciement à titre d'unique réparation du préjudice subi.

En effet, la loi n'a prévu la réintégration du salarié que dans le cas de la nullité du licenciement, si la demande est fondée, sauf si lors de l'exécution du jugement celui-ci opte pour l'indemnisation. Depuis plusieurs années, les juges ont élargi les cas pour lesquels il convient de procéder à la réintégration.

\section{II - CRITÈRES JUDICIAIRES EN MATIÈRE DE SÉCURITÉ DE L'EMPLOI}

Depuis 2002, le Tribunal constitutionnel a instauré une règle selon laquelle il convient de procéder à la réintégration du salarié en cas de licenciement portant atteinte aux droits fondamentaux, que ce soit du fait du licenciement lui-même ou du fait de la forme ou de la procédure de licenciement.

Dans le premier cas, le juge a considéré qu'il existe, en plus du licenciement nul, le licenciement sans motif valable et le licenciement frauduleux. Le licenciement nul est constaté lorsqu'aucun motif n'est invoqué pour justifier le licenciement (qu'il s'agisse d'un licenciement notifié par écrit, d'un licenciement oral ou d'un licenciement de fait). II en va de même en cas d'« expiration » d'un contrat à durée déterminée ou d'« achèvement » d'un contrat de louage de services qui serait dénaturé (un contrat simulé ou frauduleux ne peut prendre fin qu'en raison d'un motif valable). Un licenciement frauduleux se caractérise par l'imputation de faits notoirement inexistants, faux ou imaginaires, par l'attribution d'une faute non prévue légalement ou par l'obligation, sur pression, de renoncer à des preuves ou d'en fabriquer.

Dans le deuxième cas, la forme et la procédure du licenciement peuvent permettre de demander la réintégration lorsqu'il y a violation de la procédure régulière et du droit de la défense (quand la procédure prévue par la loi n'est pas respectée ou que les faits constituant la faute ne sont pas identifiés dans la lettre de notification, par exemple).

Ainsi, ce sont les salariés qui ont finalement le choix de décider si les indemnités payées par leur employeur constituent une réparation suffisante au regard de la violation de leurs droits, ou s'ils demandent leur réintégration en avançant, comme l'a soutenu le Tribunal constitutionnel, que "l'extinction unilatérale de la relation de travail, fondée uniquement et exclusivement sur la volonté de l'employeur, se trouve frappée de nullité - et que par conséquent le licenciement ne produira aucun effet juridique - quand elle se produit avec une violation des droits fondamentaux de la personne $»^{2}$.

2 Fondement juridique 4 de l'arrêt du Tribunal constitutionnel rendu dans le Dossier 2371-2004-AA/TC. 
Si l'employeur décide de licencier le salarié et de payer les indemnités prévues par la loi, c'est le salarié qui aura le choix d'accepter ce paiement ou de le refuser, afin de demander ensuite sa réintégration. Le Tribunal constitutionnel a établi que si le salarié perçoit les indemnités, il ne pourra plus réclamer sa réintégration.

\section{III - TRAITEMENT DES SALARIÉS DE DIRECTION ET DE CONFIANCE}

Conformément aux normes de la LPCT susmentionnées, si le licenciement d'un salarié de direction ou de confiance est déclaré nul, le salarié peut demander sa réintégration; si son licenciement est arbitraire, il a droit aux indemnités de licenciement; c'est dans le seul cas où le licenciement se produit pour un motif signalé dans la loi (la même que celle qui n'a pas inclus en tant que telle la perte de confiance), dûment contrôlé, que le salarié n'a pas droit aux indemnités. Cependant, en conséquence de critères jurisprudentiels adoptés lors de l'examen de cas impliquant des salariés de direction ou de confiance, le niveau de protection de ces derniers a diminué.

Lorsqu'au début de la décennie précédente, le Tribunal constitutionnel a commencé à ordonner la réintégration de salariés, il a également défini le critère applicable aux salariés de direction et de confiance, bien que ce critère soit variable. Au départ, la possibilité de réintégration leur a été refusée (ils avaient droit aux indemnités de licenciement), le critère ayant ensuite été modifié par la prise en compte de la manière dont ils ont accédé au poste. Dans le cas des salariés qui ont été embauchés pour occuper un poste de direction ou de confiance, il a établi qu'ils ont droit aux indemnités prévues par la loi; tandis que dans le cas des salariés qui ont été promus à ce poste, ils peuvent choisir entre la réintégration au poste antérieur et les indemnités de licenciement ${ }^{3}$.

Ce dernier critère est également celui que la Cour suprême appliquait depuis la précédente décennie. Cependant, en 2016 la Cour a changé radicalement de position. Lors de la décision Cassation 18450-2015-Lima, la Haute juridiction a considéré qu'un salarié embauché à un poste de confiance ayant pris fin pour perte de confiance, n'a pas droit aux indemnités pour licenciement arbitraire prévues par la loi. Même si elle est revenue par la suite à son critère antérieur dans d'autres cas, ce retour n'a pas été définitif.

En 2017, lors de la Séance plénière des juridictions syndicales de district de Lima il a arrêté, à la majorité des votes, qu'il n'y a pas lieu de verser les indemnités pour licenciement arbitraire dans le cas des salariés de confiance exclusive (ceux qui ont été embauchés à ce poste), car, conformément au deuxième paragraphe de l'article $44^{\circ}$ de la LPCT, tant au moment de la nomination qu'au moment de la promotion du salarié, la loi ne protège pas celui-ci de l'abus de droit ou de la simulation. Et en 2018 le même critère a été repris lors de la Vllème Séance plénière des juridictions suprêmes.

Avec ce critère, les membres de la Cour supérieure de Lima dans un premier temps, et les magistrats de la Cour suprême dans un deuxième, ont donné leur position en faveur de la perte de confiance comme motif juste de licenciement - ne donnant pas droit à des indemnités de licenciement - pour le cas des salariés directement embauchés pour

3 Par la suite, dans le cas de salariés du secteur public, le Tribunal constitutionnel a considéré que la perte de confiance était un motif de licenciement ne donnant pas droit au paiement d'indemnités, mais uniquement à la réintégration dans le cas où le salarié aurait été promu à un poste de confiance, hypothèse dans laquelle la réintégration se ferait au poste occupé avant la promotion. 
occuper des postes de direction ou de confiance. Cependant, il convient d'exprimer des préoccupations concernant le critère utilisé.

Les salariés de direction et de confiance ont également droit à une protection adéquate contre le licenciement arbitraire garanti par l'article 27 de la Constitution. II n'est pas légitime que ces salariés soient privés du droit de recevoir des indemnités de licenciement. La «perte de confiance» ne constitue pas un motif valable de licenciement reconnu par la loi. Il s'agit d'une mesure qui dépend exclusivement de la volonté de l'employeur qui peut l'utiliser y compris de façon arbitraire, dans le but de mettre fin à la relation de travail d'un salarié sans obligation de verser le moindre paiement supplémentaire pour ce motif.

Il ne nous paraît pas approprié d'assimiler la position des salariés du secteur privé qui occupent des postes de direction ou de confiance et qui ont été embauchés pour occuper ces postes, à ce qui se passe pour les fonctionnaires de l'État librement nommés et congédiés, comme les ministres ou leurs conseillers, qui n'ont manifestement pas droit à la sécurité du travail.

Le fait que le Tribunal constitutionnel et la Cour suprême aient adopté une position admettant la réintégration dans des hypothèses non prévues par la loi, en se basant sur des normes de niveau constitutionnel, a entraîné finalement la privation de protection pour un groupe de salariés (le personnel de direction et de confiance), alors que les traités internationaux et la Constitution ne les excluent pas du droit à la sécurité de l'emploi. 